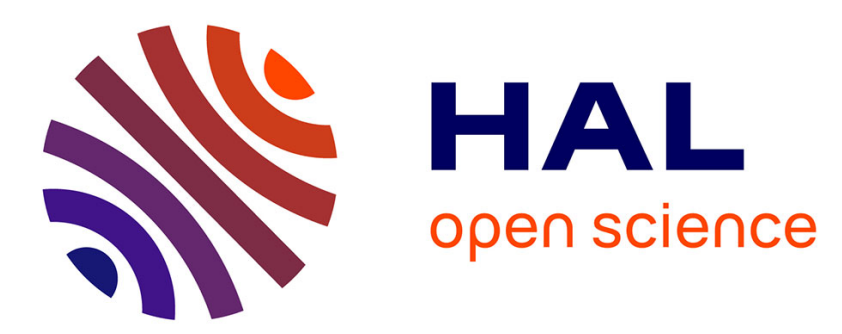

\title{
Ionization energy of donor and acceptor impurities in semiconductor nanowires: importance of dielectric confinement
}

\author{
Mamadou Diarra, Yann-Michel Niquet, Christophe Delerue, Guy Allan
}

\section{To cite this version:}

Mamadou Diarra, Yann-Michel Niquet, Christophe Delerue, Guy Allan. Ionization energy of donor and acceptor impurities in semiconductor nanowires: importance of dielectric confinement. Physical Review B: Condensed Matter and Materials Physics (1998-2015), 2007, 75, pp.045301. 10.1103/PhysRevB.75.045301 . hal-00283119

\section{HAL Id: hal-00283119 \\ https://hal.science/hal-00283119}

Submitted on 6 Aug 2021

HAL is a multi-disciplinary open access archive for the deposit and dissemination of scientific research documents, whether they are published or not. The documents may come from teaching and research institutions in France or abroad, or from public or private research centers.
L'archive ouverte pluridisciplinaire HAL, est destinée au dépôt et à la diffusion de documents scientifiques de niveau recherche, publiés ou non, émanant des établissements d'enseignement et de recherche français ou étrangers, des laboratoires publics ou privés. 


\title{
Ionization energy of donor and acceptor impurities in semiconductor nanowires: Importance of dielectric confinement
}

\author{
Mamadou Diarra, ${ }_{1}^{1}$ Yann-Michel Niquet, ${ }^{2}$ Christophe Delerue, ${ }^{1}$ and Guy Allan ${ }^{1}$ \\ ${ }^{1}$ Institut d'Electronique, de Microélectronique et de Nanotechnologie (UMR CNRS 8520), Département ISEN, 41 boulevard Vauban, \\ F-59046 Lille Cedex, France \\ ${ }^{2}$ Département de Recherche Fondamentale sur la Matière Condensée, SP2M/L_Sim, CEA Grenoble, 38054 Grenoble Cedex 9, France
}

(Received 17 July 2006; revised manuscript received 4 October 2006; published 3 January 2007)

\begin{abstract}
Calculations of the electronic states of donor and acceptor impurities in nanowires show that the ionization energy of the impurities is strongly enhanced with respect to the bulk, above all when the wires are embedded in a material with a low dielectric constant. In free-standing nanowires with diameter below $10 \mathrm{~nm}$, the ionization of the impurities at $300 \mathrm{~K}$ is strongly reduced and heavy doping is necessary to obtain conductive systems. These results imply that the critical density for metal-nonmetal transitions is not the same as in the bulk. Experiments are proposed to test the predictions.
\end{abstract}

DOI: 10.1103/PhysRevB.75.045301

PACS number(s): 73.22.-f, 73.63.Nm

\section{INTRODUCTION}

Recent breakthroughs in the growth of semiconductor nanowires (SNW's) have opened up great opportunities for nanoscale device applications, ${ }^{1-4}$ increasing the interest in new one-dimensional (1D) nanostructures whose properties may differ from those of carbon nanotubes. One main advantage of SNW's with respect to carbon nanotubes is that they remain semiconducting independent of their diameter and orientation, giving the ability to control their properties by doping. ${ }^{5}$ The availability of both $n$ - and $p$-type semiconductor components is also important for the realization of nanowire-based electronics. ${ }^{1,6}$ Therefore a large number of experimental works have addressed the problem of doping $^{6-10}$ and of its modulation ${ }^{5,11}$ in SNW's. While there is no doubt that $p$ - and $n$-type SNW's can be produced, the question of how their electrical conductivity depends on the doping level remains largely open. Most of the works showing good transport properties concern SNW's doped with high impurity concentration, near or above the Mott density corresponding to the metal-nonmetal transition in the bulk semiconductor. $^{7-9,12,13}$ Otherwise, there are indications that the conductivity of the SNW's is extremely low. ${ }^{7}$

Thus the problem of the doping efficiency must be investigated for SNW's. In a recent paper, Fernández-Serra et al. ${ }^{14}$ have shown that the impurities become electrically inactive after segregation at the surface of the SNW's. Here we consider the electronic structures of impurities located in the core of the SNW's. In bulk semiconductors, donor and acceptor impurities have been extensively investigated within the envelope function approach, ${ }^{15}$ leading to the following qualitative picture. The Coulomb potential of the impurity nucleus gives rise to bound states in the energy gap. But in conventional semiconductors, this potential is strongly screened (dielectric constant $\epsilon \approx 10-20$ ), the Bohr radius is large $(\approx 1-5 \mathrm{~nm})$, and the ionization energy is only of a few hundredths of an electron volt so that the impurities are ionized at room temperature.

In this paper, we show that this simple picture often breaks down in SNW's because the ionization energy of the impurities is greatly enhanced. It is already well known that, when the thickness of a wire becomes of the order of the impurity Bohr radius, the ionization energy increases due to the quantum confinement (it even tends to infinity in the strictly 1D limit). ${ }^{16,17}$ But experimentally, wire diameters are mostly in the 10 -nm range and thus the quantum confinement effect is weak. Nevertheless, we still predict that the ionization energy is enhanced due to the so-called dielectric confinement. ${ }^{18}$ This effect occurs when there is an important dielectric mismatch between the wire and its surroundings, which is often the case experimentally. In that case, we obtain that the doping efficiency of the impurities at room temperature is considerably reduced for SNW diameters below $10 \mathrm{~nm}$. Then it is necessary to use heavy doping to obtain good electrical properties.

\section{METHODOLOGY}

We calculate the electronic states of dopants in Si nanowires (SiNW's) using periodic boundary conditions along the nanowire axis. The length of the 1D supercell, which contains one dopant, is adjusted so that the dispersion of the impurity band is below $0.1 \mathrm{meV}$. The supercell actually contains up to $1.3 \times 10^{5}$ atoms due to the large Bohr radius of the impurities. To deal with this complex problem, we use the tight binding (TB) method because it allows the study of shallow impurities in bulk semiconductors, as shown recently by Martins et al., ${ }^{19}$ and because it is very well adapted to semiconductor nanostructures, ${ }^{18}$ including SNW's. ${ }^{20}$ The TB Hamiltonian is written as

$$
H=H_{0}+U_{\text {imp }}+\Sigma,
$$

where $H_{0}$ is the Hamiltonian of the perfect wire in which all the dangling bonds at the surface are saturated by hydrogen atoms. ${ }^{20}$ We use here the $s p^{3} d^{5} s^{*}$ model of Ref. 21 but similar results are obtained with the $s p^{3}$ Hamiltonian of Ref. 22. Spin-orbit coupling is included. The term $U_{\mathrm{imp}}= \pm V\left(\mathbf{r}, \mathbf{r}_{0}\right)$ in Eq. (1) describes the potential induced by the impurity located at $\mathbf{r}_{0}$. Here $V\left(\mathbf{r}, \mathbf{r}^{\prime}\right)$ is the energy of a charge $+e$ at $\mathbf{r}$ in the electrostatic potential created by a charge $+e$ at $\mathbf{r}^{\prime}$, and the sign $+(-)$ holds for an acceptor (donor). $V$ is the solu- 
tion of the Poisson equation in which the system is represented by a cylinder of radius $R$ and of dielectric constant $\epsilon_{\text {in }}$ embedded in a medium of dielectric constant $\epsilon_{\text {out }}$. We use the bulk experimental value $\epsilon_{\mathrm{in}}=11.7$. This approach is fully justified when $R \geq 2 \mathrm{~nm} \cdot{ }^{18,23}$ Details of the TB method and of the calculation of $V$ are given in Ref. 20.

Screening properties are drastically different in 1D and in 3D. In a bulk semiconductor (3D), the screening of a nucleus charge $+e$ leaves a total charge $+e / \epsilon_{\text {in }}$ at the impurity site, the remaining charge $+e\left(1-1 / \epsilon_{\text {in }}\right)$ being repelled at infinity. Thus the bulk potential is $V_{\mathrm{b}}\left(\mathbf{r}, \mathbf{r}_{0}\right)=e^{2} /\left[\epsilon_{\mathrm{in}}\left|\mathbf{r}-\mathbf{r}_{0}\right|\right]$. In a SNW (1D), the charge $+e\left(1-1 / \epsilon_{\text {in }}\right)$ is repelled at the surface of the nanowire, in the vicinity of the impurity, which leads to an extra term in the potential, $V=V_{\mathrm{b}}+V_{\mathrm{s}}$. Physically, $V_{\mathrm{s}}$ is at the origin of the so-called image force in the case of planar interfaces. ${ }^{18}$ The total charge seen far from the impurity $\left(\left|\mathbf{r}-\mathbf{r}_{0}\right| \gg R\right)$ is $+e$, and thus the potential is asymptotically unscreened $\left(V \approx e^{2} /\left|\mathbf{r}-\mathbf{r}_{0}\right|\right)$.

The treatment of $U_{\text {imp }}$ (or $V$ ) in TB is straightforward. As a slowly variable potential, it only appears in the diagonal of the Hamiltonian matrix. ${ }^{18}$ At the impurity site, we write $V_{\mathrm{b}}\left(\mathbf{r}_{0}, \mathbf{r}_{0}\right)=V_{0}$ where $V_{0}$ is a parameter describing central cell effects characteristic of the impurity. ${ }^{15}$ According to Ref. 19, we determine the value of $V_{0}$ so that the ionization energy of the impurity is in good agreement with the experimental value in the bulk situation. We calculate an effective Bohr radius as $r_{\mathrm{B}}=\sqrt{\left\langle\left|\mathbf{r}-\mathbf{r}_{0}\right|^{2}\right\rangle}$ where the average is taken over the impurity state. We obtain in the bulk $r_{\mathrm{B}}=2.69 \mathrm{~nm}$ for $\mathrm{P}$, $2.21 \mathrm{~nm}$ for As, and $3.18 \mathrm{~nm}$ for $\mathrm{Sb}$.

The last term $\Sigma$ in the TB Hamiltonian [Eq. (1)] is the self-energy potential which represents the interaction between the carrier (electron or hole) and the surface polarization charges which are induced by its own presence. As discussed in Refs. 18, 20, and 24, it can be written as $\Sigma(\mathbf{r})= \pm \frac{1}{2} V_{\mathrm{s}}(\mathbf{r}, \mathbf{r})(+$ for an electron, - for a hole). The factor $1 / 2$ follows from the adiabatic building of the charge distribution..$^{18}$ This description of the quasiparticle spectrum of nanostructures is justified by $G W$ calculations on SiNWs (Ref. 25) and on Si nanocrystals. ${ }^{26}$ For a free electron in the lowest conduction state $|\psi\rangle$ of a SNW, the main effect of $\Sigma$ is a rigid upward shift of its energy (the so-called self-energy correction) with respect to the single-particle energy of the TB Hamiltonian $H_{0}$. This shift $\langle\Sigma\rangle$ is equal to $\left\langle\psi\left|V_{\mathrm{s}}(\mathbf{r}, \mathbf{r})\right| \psi\right\rangle / 2$ where $^{20}$

$$
\left\langle\psi\left|V_{\mathrm{s}}(\mathbf{r}, \mathbf{r})\right| \psi\right\rangle=\frac{2 e^{2}}{\epsilon_{\text {in }} R} \frac{\epsilon_{\text {in }}-\epsilon_{\text {out }}}{\epsilon_{\text {in }}+\epsilon_{\text {out }}} F\left(\frac{\epsilon_{\text {in }}}{\epsilon_{\text {out }}}\right),
$$

in which $F$ is a function given in Ref. 20.

\section{RESULTS AND DISCUSSIONS}

We present in Fig. 1 the ionization energy $E_{\mathrm{I}}$ of donor impurities located at the center of free-standing $\langle 100\rangle$ oriented SiNW's $\left(\epsilon_{\mathrm{out}}=1\right) . E_{\mathrm{I}}$ is given by the difference between the band edge of the perfect wire and the lowestenergy state of the impurity. There is some dependence of the results on the nature of the impurity (chemical effects) but the overall behavior is the same for $\mathrm{P}$, As, and $\mathrm{Sb}$. High

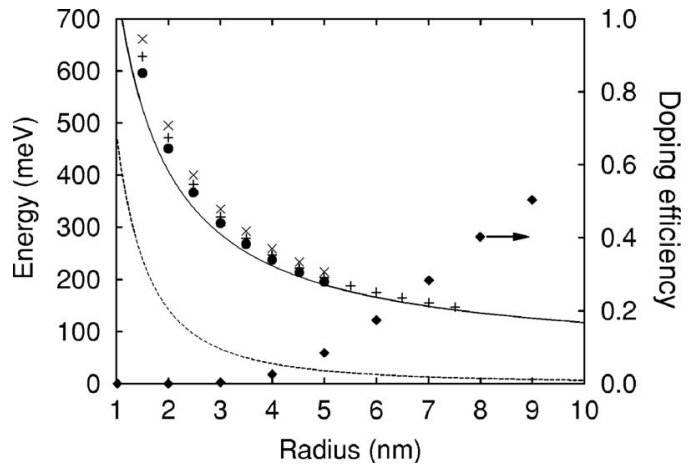

FIG. 1. Ionization energy $E_{\mathrm{I}}$ versus the wire radius $R$ for donor impurities $(\mathrm{P}=+, \mathrm{As}=\times, \mathrm{Sb}=\mathbf{O})$ located along the axis of a freestanding $\operatorname{SiNW}\left(\epsilon_{\text {out }}=1\right)$. The bulk values $E_{\mathrm{I}}^{0}$ are equal to $45 \mathrm{meV}$ for $\mathrm{P}, 54 \mathrm{meV}$ for $\mathrm{As}$, and $39 \mathrm{meV}$ for $\mathrm{Sb}$. The solid curve represents Eq. (3) for a P impurity. Dashed line: shift of the conduction band edge due to quantum confinement. Doping efficiency $(\diamond)$ : probability of ionization towards the conduction band for a $\mathrm{P}$ impurity $(300 \mathrm{~K})$.

values of $E_{\mathrm{I}}$ are predicted, even for large SiNW's $(R>5 \mathrm{~nm})$ in which the effect of quantum confinement is weak. In particular, the enhancement of $E_{\mathrm{I}}$ is much larger than the shift of the conduction band edge due to quantum confinement (dashed line in Fig. 1). This is due to the interaction $\left[\propto\right.$ the image charge potential $V_{\mathrm{s}}\left(\mathbf{r}, \mathbf{r}_{0}\right)$ ] between the electron and the surface polarization charge $+e\left(1-1 / \epsilon_{\text {in }}\right)$ induced by the impurity nucleus.

To prove this interpretation, we can estimate the contributions of $U_{\text {imp }}$ and $\Sigma$ to the variation of $E_{\mathrm{I}}$ with respect to the bulk value $E_{\mathrm{I}}^{0}$. The contribution of the image charge potential in $U_{\text {imp }}$ is $\left\langle\psi_{\text {imp }}\left|V_{\mathrm{s}}\left(\mathbf{r}, \mathbf{r}_{0}\right)\right| \psi_{\text {imp }}\right\rangle$, where $\left|\psi_{\text {imp }}\right\rangle$ is the bound state. $V_{\mathrm{s}}\left(\mathbf{r}, \mathbf{r}_{0}\right)$ is slowly varying if $\left|\mathbf{r}-\mathbf{r}_{0}\right| \ll R$ and $\left|\mathbf{r}_{0}\right| \ll R$. In the limit of thick wires $\left(r_{\mathrm{B}} \ll R\right)$, we can therefore replace $\mathbf{r}_{0}$ by $\mathbf{r}$. Moreover, as shown in Ref. $20, V_{\mathrm{s}}(\mathbf{r}, \mathbf{r})$ is almost constant in the region where both $\psi_{\text {imp }}(\mathbf{r})$ and the free electron wave function $\psi(\mathbf{r})$ have non-negligible amplitudes. Thus, we can write $\left\langle\psi_{\text {imp }}\left|V_{\mathrm{s}}\left(\mathbf{r}, \mathbf{r}_{0}\right)\right| \psi_{\text {imp }}\right\rangle$ $\approx\left\langle\psi_{\text {imp }}\left|V_{\mathrm{s}}(\mathbf{r}, \mathbf{r})\right| \psi_{\text {imp }}\right\rangle \approx\left\langle\psi\left|V_{\mathrm{s}}(\mathbf{r}, \mathbf{r})\right| \psi\right\rangle$. The self-energy corrections to $|\psi\rangle$ and $\left|\psi_{\text {imp }}\right\rangle$ are, moreover, almost the same, so that their contributions cancel in the ionization energy (the electron probes almost the same self-energy potential when it is bound or free). Thus we deduce from Eq. (2) that

$$
E_{\mathrm{I}}-E_{\mathrm{I}}^{0} \approx \frac{2 e^{2}}{\epsilon_{\text {in }} R} \frac{\epsilon_{\text {in }}-\epsilon_{\text {out }}}{\epsilon_{\text {in }}+\epsilon_{\text {out }}} F\left(\frac{\epsilon_{\text {in }}}{\epsilon_{\text {out }}}\right) \propto \frac{1}{R} .
$$

Figure 1 shows that $E_{\mathrm{I}}$ deduced from Eq. (3) is close to the TB results which allows one to conclude that dielectric effects have a huge influence on the ionization energy of impurities in SiNW's. Using $E_{\mathrm{I}}$ and the density of states in SiNW's ${ }^{20}$ we estimate that the doping efficiency (probability of ionization) at $300 \mathrm{~K}$ of a $\mathrm{P}$ impurity at the center of a SiNW is below $50 \%$ for $R<9 \mathrm{~nm}$, below $10 \%$ for $R<4 \mathrm{~nm}$, and below $0.1 \%$ for $R<2 \mathrm{~nm}$ (Fig. 1).

Equation (3) is only valid when $R \gg r_{\mathrm{B}}$; otherwise, quantum confinement cannot be neglected. To evaluate its effects, we have calculated the ionization energy when $\epsilon_{\text {out }}=\epsilon_{\text {in }}$-i.e., 


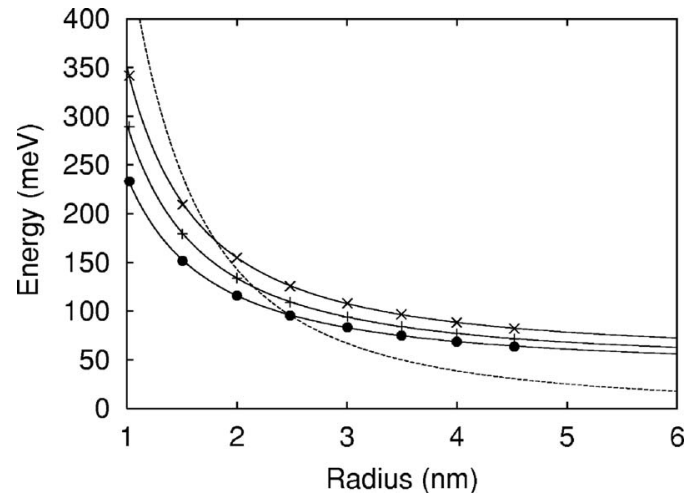

FIG. 2. Same as Fig. 1 but for $\epsilon_{\mathrm{out}}=\epsilon_{\text {in }}(\mathrm{P}=+, \mathrm{As}=\times, \mathrm{Sb}=\bullet)$. Curves: fit of the TB values with $E_{\mathrm{I}}^{0}+a\left(r_{\mathrm{B}} / R\right)^{b}(\mathrm{P}, a=57.33 \mathrm{meV}$, $b=1.47$; As, $a=86.32 \mathrm{meV}, b=1.55$; Sb, $a=40.60 \mathrm{meV}, b=1.37$ ). Dashed line: shift of the conduction band edge due to quantum confinement.

without dielectric confinement $\left(V_{\mathrm{s}}=0\right)$. We obtain that $E_{\mathrm{I}}$ is also enhanced but to lesser extent (Fig. 2). $E_{\mathrm{I}}-E_{\mathrm{I}}^{0}$ is now smaller than the shift of the conduction band induced by the quantum confinement, and it follows a $1 / R^{b}$ law $(b \approx 1.4)$ instead of $1 / R$ in the previous situation [Eq. (3)]. Thus it is possible to vary considerably the ionization energy of impurities and the electrical properties in SNW's by playing on their dielectric environment ${ }^{24}$ which could be easily tested experimentally. In particular, the ionization energy must be considerably reduced in SNW's surrounded by a metallic gate or a high- $k$ dielectric material. Furthermore, the embedding of SNW's in solvents with an important frequency dependence of the dielectric constant could lead to very interesting phenomena as shown by Chazalviel et al. ${ }^{24}$

The confinement has also an influence on the bound wave function (Fig. 3): its extension is reduced when decreasing $R$, and it is also deformed, being more elongated along the axis of the wire than in the transverse directions. The confinement effect could be probed by measuring the hyperfine structure of electron spin resonance ${ }^{27}$ since the hyperfine splitting is proportional to the weight $|\Psi(0)|^{2}$ of the wave function on

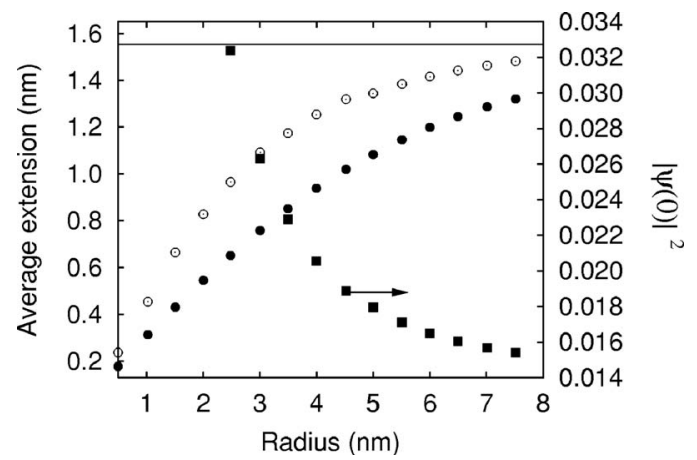

FIG. 3. Average extensions $\sqrt{\left\langle z^{2}\right\rangle}(\odot)$ and $\sqrt{\left\langle x^{2}\right\rangle}(\bullet)$ of the bound state of a $\mathrm{P}$ impurity located along the axis $z$ of a free-standing SiNW $\left(\epsilon_{\text {out }}=1\right)$ as a function of its radius $R$. The horizontal line represents the bulk limit $\left(r_{\mathrm{B}} / \sqrt{3}\right)$ equal to $1.55 \mathrm{~nm}$. Weight $|\Psi(0)|^{2}$ of the wave function on the $\mathrm{P}$ atom, the bulk value being equal to $0.014(\square)$

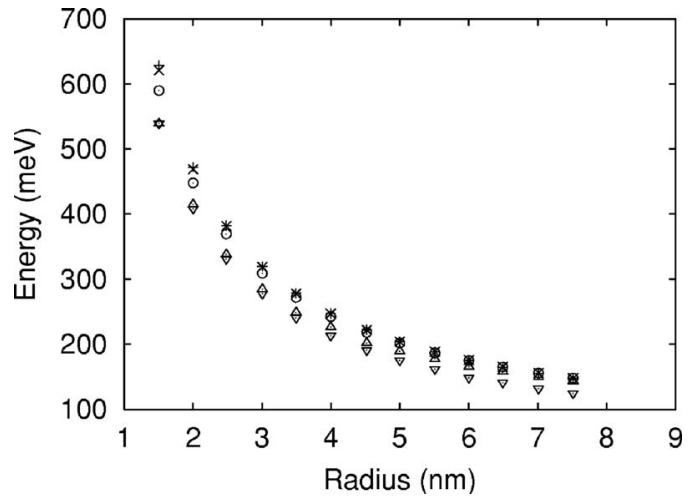

FIG. 4. Ionization energy versus the radius $R$ for a $\mathrm{P}$ impurity located at different distances $r=0(+), r=0.25 R(\times), r=0.5 R(\odot)$, $r=0.75 R(\Delta)$ from the axis of a free-standing $\operatorname{SiNW}\left(\epsilon_{\text {out }}=1\right)$. The symbols $\nabla$ correspond to an impurity located at a fixed distance $(0.68 \AA)$ from the surface.

the impurity atom. Figure 3 shows that $|\Psi(0)|^{2}$ increases when going to small $R$. The enhancement of the hyperfine splitting for $\mathrm{P}$ impurities was observed experimentally in $\mathrm{Si}$ nanocrystals and was explained by the quantum confinement. ${ }^{28}$

The importance of dielectric effects on impurities in SNW's is a general result when $\epsilon_{\text {in }} \gg \epsilon_{\text {out }}$. Figure 4 shows that $E_{\mathrm{I}}$ has a weak dependence on the impurity position in the SiNW, which is due to the flatness of the image charge potential in the wire. Similar results are obtained for acceptor impurities (Fig. 5), for $\langle 110\rangle$ - and $\langle 111\rangle$-oriented SiNW's and for SNW's made from other semiconductors. Obviously, the conclusions of our work concern impurities which can be considered as independent. Since the extension of the wave function decreases when going to smaller wires, we could consider at first glance that the Mott density is higher in SNW's than in the bulk semiconductor. But we have to take care that in these systems Coulomb interactions decay more slowly than in bulk because they are unscreened at large distance. Therefore, the nature of the excited states, the coupling between neighbor impurity states, and the dependence of the efficiency of ionization on the impurity concentration become considerably more complex problems than in the

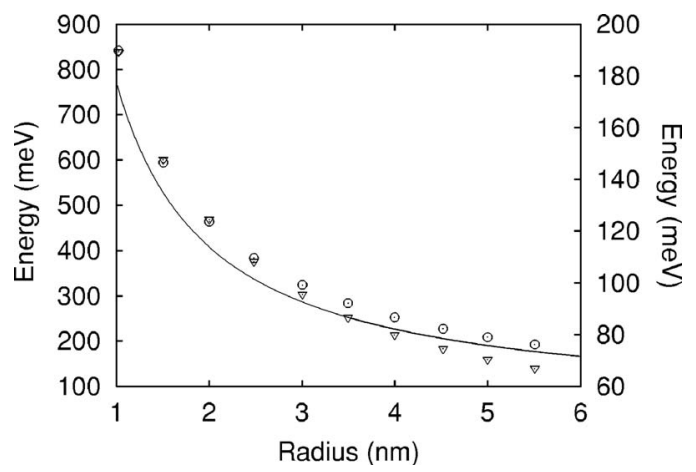

FIG. 5. Ionization energy versus the wire radius $R$ for a B impurity (acceptor) located along the axis of a SiNW for $\epsilon_{\text {out }}=1(\odot$, left axis) and for $\epsilon_{\text {out }}=\epsilon_{\text {in }}(\nabla$, right axis). The solid curve corresponds to [Eq. (3)] for $E_{\mathrm{I}}^{0}=45 \mathrm{meV}$ and $\epsilon_{\text {out }}=1$. 
bulk. Criteria for metal-nonmetal transition must be revised in SNW's.

\section{CONCLUSIONS}

TB calculations including self-energy corrections have been performed on acceptor and donor impurities in SNW's. Their electronic structure strongly depends on the diameter of the wires and on their dielectric surrounding. In freestanding SNW's, the ionization energy can be enhanced in such a way that thermal ionization of the carriers becomes unlikely. Therefore, good electrical properties require heavy doping, beyond a critical density which must depend on the wire diameter and on its environment. These effects due to the dielectric confinement, and to a lesser extent to quantum confinement, could be probed by transport measurements and by electron spin resonance, at varying temperature, SNW radius, and doping density. The nature of the metalnonmetal transition is not the same in SNW's as in the bulk, and many-body calculations are needed to deal with the many-impurity problem.

\section{ACKNOWLEDGMENTS}

This work is supported by the French "Action Concertée Incitative" (ACI) "Transnanofils." Y.M.N. acknowledges support from the European Integrated Project (IP) NODE (EU Contract No. 015783 NODE) and C.D. from the Université Catholique de Lille.
${ }^{1}$ C. M. Lieber, Mater. Res. Bull. 28, 486 (2003).

${ }^{2}$ P. Yang, Mater. Res. Bull. 30, 85 (2005).

${ }^{3}$ C. Thelander, H. A. Nilsson, L. E. Jensen, and L. Samuelson, Nano Lett. 5, 635 (2005).

${ }^{4}$ Y. Cui, Z. H. Zhong, D. L. Wang, W. U. Wang, and C. M. Lieber, Nano Lett. 3, 149 (2003).

${ }^{5}$ C. Yang, Z. Zhong, and C. M. Lieber, Science 310, 1304 (2005).

${ }^{6}$ A. B. Greytak, L. J. Lauhon, M. S. Gudiksen, and C. M. Lieber, Appl. Phys. Lett. 84, 4176 (2004).

${ }^{7}$ Y. Cui, X. Duan, J. Hu, and C. M. Lieber, J. Phys. Chem. B 104, 5213 (2000).

${ }^{8}$ J.-Y. Yu, S.-W. Chung, and J. R. Heath, J. Phys. Chem. B 104, 11864 (2000).

${ }^{9}$ K.-K. Lew, L. Pan, T. E. Bogart, S. M. Dilts, E. C. Dickey, J. M. Redwing, Y. Wang, M. Cabassi, T. S. Mayer, and S. W. Novak, Appl. Phys. Lett. 85, 3101 (2004).

${ }^{10}$ K. Byon, D. Tham, J. E. Fischer, and A. T. Johnson, Appl. Phys. Lett. 87, 193104 (2005).

${ }^{11}$ M. S. Gudiksen, L. J. Lauhon, J. Wang, D. Smith, and C. M. Lieber, Nature (London) 415, 617 (2002).

${ }^{12}$ G. Zheng, W. Lu, S. Jin, and C. M. Lieber, Adv. Mater. (Weinheim, Ger.) 16, 1890 (2004).

${ }^{13}$ G. Cheng, E. Stern, D. Turner-Evans, and M. Reed, Appl. Phys. Lett. 87, 253103 (2005).

${ }^{14}$ M. V. Fernández-Serra, Ch. Adessi, and X. Blase, Phys. Rev. Lett.
96, 166805 (2006).

${ }^{15}$ W. Kohn and J. M. Luttinger, Phys. Rev. 98, 915 (1955).

${ }^{16}$ R. Loudon, Am. J. Phys. 27, 649 (1959).

${ }^{17}$ G. W. Bryant, Phys. Rev. B 29, 6632 (1984).

${ }^{18} \mathrm{C}$. Delerue and M. Lannoo, Nanostructures-Theory and Modelling (Springer-Verlag, Berlin, 2004).

${ }^{19}$ A. S. Martins, J. G. Menchero, R. B. Capaz, and B. Koiller, Phys. Rev. B 65, 245205 (2002).

${ }^{20}$ Y. M. Niquet, A. Lherbier, N. H. Quang, M. V. Fernández-Serra, X. Blase, and C. Delerue, Phys. Rev. B 73, 165319 (2006).

${ }^{21}$ T. B. Boykin, G. Klimeck, and F. Oyafuso, Phys. Rev. B 69, 115201 (2004).

${ }^{22}$ Y. M. Niquet, C. Delerue, G. Allan, and M. Lannoo, Phys. Rev. B 62, 5109 (2000).

${ }^{23}$ C. Delerue, M. Lannoo, and G. Allan, Phys. Rev. B 68, 115411 (2003).

${ }^{24}$ J.-N. Chazalviel, F. Ozanam, and V. M. Dubin, J. Phys. I 4, 1325 (1994).

${ }^{25}$ C. Delerue, Y. M. Niquet, and G. Allan (unpublished).

${ }^{26}$ C. Delerue, M. Lannoo, and G. Allan, Phys. Rev. Lett. 84, 2457 (2000).

${ }^{27}$ G. Feher, Phys. Rev. 114, 1219 (1959).

${ }^{28}$ M. Fujii, A. Mimura, S. Hayashi, Y. Yamamoto, and K. Murakami, Phys. Rev. Lett. 89, 206805 (2002). 